\title{
Cluster Head Location based Base Station Mobility in Wireless Sensor Network
}

\author{
Monica R Mundada \\ Research Scholar \\ Dr M G R University \\ Chennai, India.
}

\author{
Pallavi B. Kamble \\ Dept of Computer Science \\ MSRIT
}

\author{
T Bhuvaneswari \\ Dept of Computer Application \\ L N Government College \\ Ponneri, India.
}

\author{
V CyrilRaj \\ Dept of CSE and IT \\ Dr M G R University, Chennai, \\ India.
}

\author{
Shubhra Kejriwal \\ Dept of Computer Science \\ MSRIT
}

\author{
Yashaswini Nag M N \\ Dept of Computer Science \\ MSRIT
}

\begin{abstract}
Wireless sensor networks (WSN) are sophisticated systems that are used to gather data from an unreachable and remote environment. WSN consists of base station and hundred to thousands of sensor nodes. The main design issues in WSN include efficient energy management and network lifetime. Most of the approaches assume the nodes to be homogenous, however they can be heterogeneous. In this paper we take a realistic approach by considering heterogeneous networks. The clustering mechanism, location of base station and the routing process are crucial to elongate the lifetime of the sensor nodes. The clusters will be formed in different topologies depending on the sensor node deployment. The mobile base station, concept of budget to the sensor nodes and multi hop routing in a sensor network with heterogeneous nodes is used to make the network energy efficient and maximize the network lifetime.
\end{abstract}

\section{General Terms}

Wireless Sensor Networks, Heterogeneous Sensor Nodes, Mobile Base Stations.

\section{Keywords}

Budget; TDMA Mobile Base Stations; Energy

Heterogeneous Nodes;

\section{INTRODUCTION}

A wireless sensor network (WSN) consists of spatially distributed autonomous sensors to monitor physicalor environmental conditions and to cooperatively pass their data through the network to a base station. Base stations collect the data from the sensor nodes.

Most research till date has been focused on networks consisting of homogenous nodes, i.e., each node is identical with respect to hardware, software and starting energy. This homogeneous architecture is attractive because it is resilient to individual failures. More recently, however, heterogeneous sensor networks have become popular, particularly in real deployments because of their potential to increase network lifetime and reliability without significantly increasing the cost.

Data transfer from the sensor node to the base station can be handled in multiple ways. These methods are normally classified into data-centric, location based and hierarchical.

In data centric methods, the base station sends a query to a certain region in the network whose data it requires. The query is sent to a random sensor node from the base station, and has to be forwarded to the intended region. The sensor nodes in the region aggregate their sensed data and route back to the base station along the reverse path discovered in the previous step.

Location based networks use location information to guide routing discovery and maintenance as well as packet forwarding, thus enabling the best routing to be selected, reducing energy consumption and optimizing the whole network.[1]

Hierarchical routing is the procedure of arranging nodes in a hierarchical manner. Each node transmits its data to its immediate superior. This process continues till the data reaches the base station. Most protocols (such as LEACH) have two levels of hierarchy. The nodes are divided into clusters based on geographical area and each cluster has a cluster head $(\mathrm{CH})$. The nodes send their data to their $\mathrm{CH}$ which then sends the data to the BS. This can be static or dynamic. In dynamic clustering in each round a different cluster head is selected.

Since Base Stations have infinite energy (are connected to a power source), new methods are being developed which allow the movement of the base stations. 
In this paper, we develop a method that combines the above approaches. We send data to the BS in rounds. In each round the heterogeneous nodes ( in terms of starting energy) that form clusters and select a cluster head using a tree based structure. Each node sends data to its $\mathrm{CH}$ using multi-hop multi-path routing. The round is divided among the clusters (using TDMA) .Each cluster gets a time slot. In each time slot, based on the cluster head of the appropriate cluster, the base station moves to an optimal location for data transfer. The $\mathrm{CH}$ then sends data to the BS either directly or using an optimal path calculated based on the characteristic distance of the node's hardware. [2]

\section{PROPOSED METHOD}

The following assumptions have been made in this paper:

1. Sensor nodes are static and heterogeneous [2] only in terms of energy.

2. Clusters have fixed $\mathrm{n}$ nodes each.

3. The set of feasible mobile base station sites is finite and known.

4. The base station has unlimited energy and only needs destination as input to move.

\subsection{Topology}

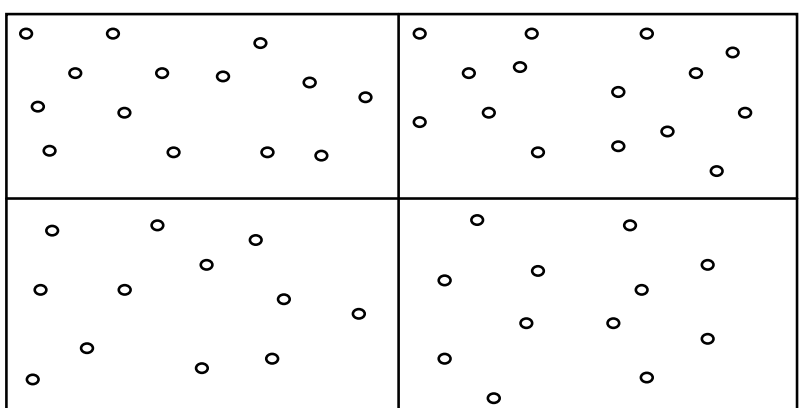

Fig 1: Clusters in Rectangular Topology

Based on the deployment of the sensor nodes they are divided into different topologies. Suppose the nodes are deployed in a rectangular fashion the cluster will be formed as shown in fig1, if deployed in a triangular fashion then fig2. The number of nodes in each cluster will be based on the following ratio,

$\mathrm{Nn}$ is the total number of nodes in the network.

$\mathrm{Nc}$ be the number of clusters then

$\mathrm{Nc}: \mathrm{Nn}=1: 25$

Considering a specific case where the total number of nodes is 100 then according to the ratio there will be 25 sensor nodes in each cluster.

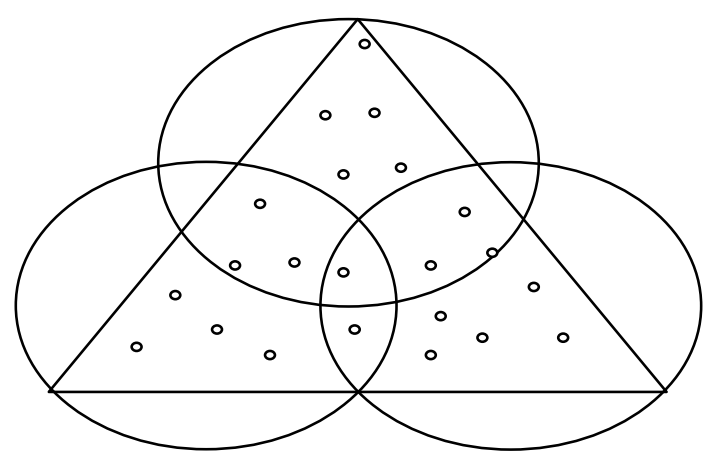

Fig 2: Clusters in Triangular Topology

\subsection{Selection of Cluster Head $(\mathrm{CH})$ :}

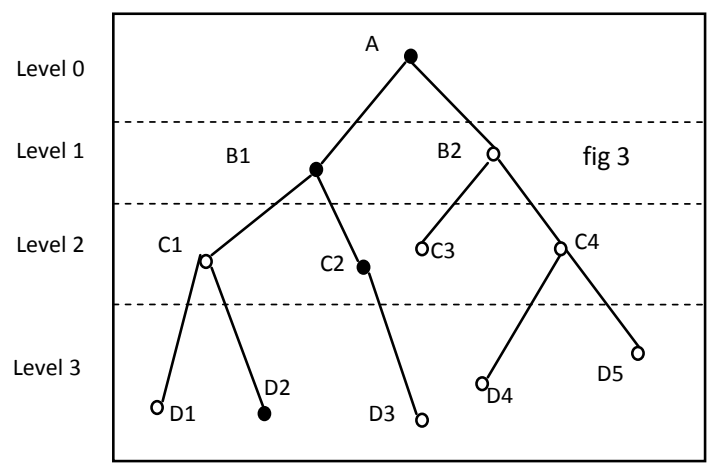

Fig 3: Budget Levels in a Cluster

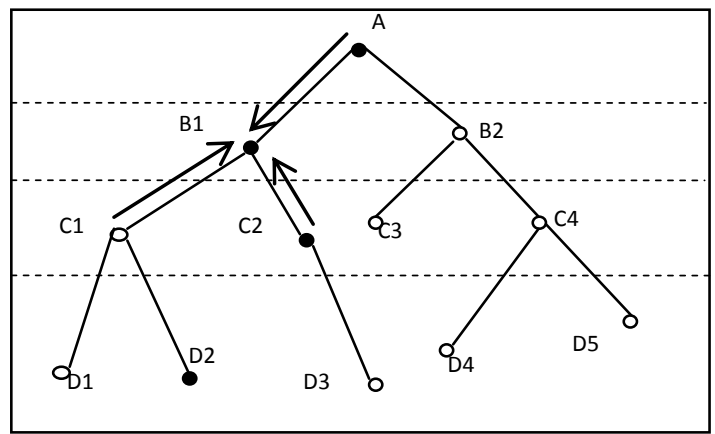

Fig 4: Nodes 1 Hop away from Cluster Head B1

In the cluster based protocol[3] the nodes in the sensor fields are grouped into clusters, and each cluster has a cluster head $(\mathrm{CH})$. All the nodes in the cluster send their data to the cluster head and cluster head then communicates with the base station. The members which are connected to the cluster head will sense the physical environment and transmit it to the cluster head by aggregating the data.

The nodes are arranged in a tree based fashion. There are nodes in each level of the tree. At every level the node with highest energy is chosen. These nodes are further compared with each other to find the node with maximum energy and is made the cluster head.

Considering one of the clusters among the formed clusters, a tree structure for the nodes would look similar to fig3.There may be $\mathrm{n}$ levels depending on the deployment of nodes. Here there are four levels from level 0 to level 3.By default node A is the node with maximum energy. At each level the node 
with maximum energy is found. Suppose, node B1 has maximum energy in level 1, node $\mathrm{C} 2$ in level 2 and node $\mathrm{D} 2$ in level 3.Now the energies of nodes A,B1,C2,D2 are compared and the node with highest energy is made the cluster head. In this way the clusters are formed with the selection of cluster head.[4]

\subsection{Communication among the Nodes (Budget)[5]:}

The sensor nodes in each cluster receives hello packet from the cluster head chosen and reply back with an acknowledgement. The acknowledgment also includes the energy of the nodes so that the cluster head can distribute its budget suitably.

The cluster head is assigned a budget of $\mathrm{n}$. It keeps one for itself and distributes the rest i.e. (n-1) among the nodes that send back the acknowledgement first. It is distributed on a first come first serve basis, assuming the nodes that come first will be at a single hop distance from the node to which it sends. The nodes that receive the budget will further distribute the budget by keeping one for it. This process continues till all the nodes get a budget of one. This budget is used to establish a communication path between the nodes on the network.

\subsubsection{Nodes with approximately Equal Energy}

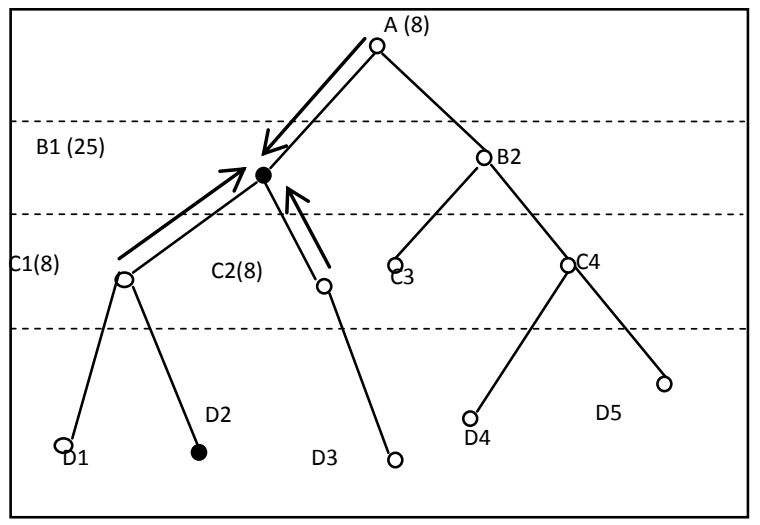

Fig 5: Equal Budget Distribution

Consider fig 5, suppose the energy of the nodes that reply back to the cluster head are comparatively same (i.e. with a difference of $15 \%$ approx), then the cluster head distributes the budget equally among the nodes. We have B1 as the cluster head having a budget of 25 among which 24 can be distributed. It receives acknowledgement from node $\mathrm{C} 1$, node $\mathrm{C} 2$ and node A, and the nodes are comparatively of same energy then each node is given a budget of 8.i.e, if $\mathrm{n}$ is the budget to be distributed among n' nodes of comparatively same energy then each of n' nodes get a budget of (n-1)/n'.

\subsubsection{Nodes with Varying Energy:}

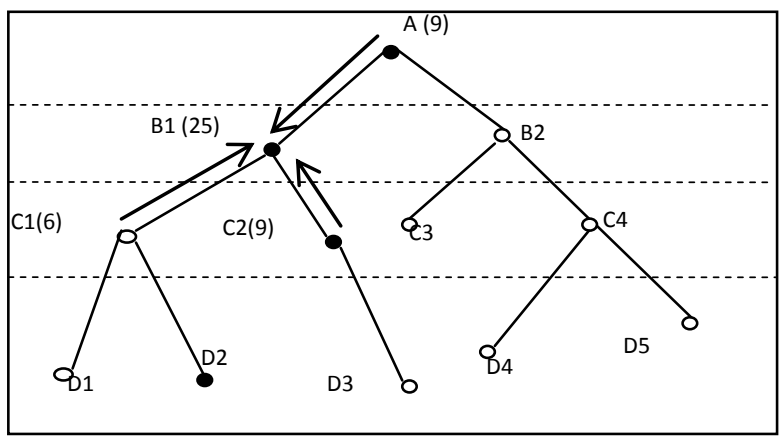

Fig 6: Unequal Budget Distribution

Now B1 being the cluster head has to distribute its budget among nodes with varying energy. Suppose the varying energy nodes are node1, node 2 and node $A$, then node A will obviously have higher energy among the three .The distribution is uneven, node A gets a more budget among the three. If $\mathrm{n}$ is the budget to be distributed among nodes $\mathrm{A}, \mathrm{B}, \mathrm{C}$ whose energy is in the order $\mathrm{A}>\mathrm{B}>\mathrm{C}$ then $\mathrm{A}$ gets a budget of $(n-1) / 2$, B gets $(n-1) / 3$ and $C$ gets $(n-1) / 4$.

\subsection{Multipath Routing:}

As mentioned earlier the budget distribution continues until each node gets a budget of 1.Once the distribution is complete, the communication path is chosen. If a node received a budget from a single node then it establishes a path with that node. Suppose it receives budget from a two or more nodes then it chooses the node with the higher energy to establish a connection, but keeps the other nodes as alternate path. Consider a scenario in fig3, if the node B2 received a hello packet from both node B1 and node $\mathrm{C} 3$ and it responds to both the nodes, then both the nodeswill give a budget to it based on its energy. Now node B1 has higher energy than node $\mathrm{C} 3$, as a result of which the node B2 makes the path through B1 as its main path and the path through the node C3 its alternate path. In this way the energy of the node is kept in mind in order to achieve maximum lifetime of the nodes and network and hence use multipath routing[6].

\subsection{Multihop Routing:}

Each node may not directly be in contact with the cluster head. In such a case the nodes make use of intermediate nodes in order to send their data to the cluster head. With the distribution of budget the communications paths were formed, which also included the alternate paths for some nodes in order to reach the cluster head. Each intermediate node aggregates the data and forwards it to the next hop until it reaches the destination i.e. the cluster head. The cluster head then aggregates the data received from all nodes and sends it to the base station. 


\subsection{Mobility of Base Stations:}

The nodes closer to the stationary base station get depleted of energy very soon as it has to aggregate all the data sent by the different cluster heads.[7] As the nodes closer to the base station keep dying, the network will lose its functionality very soon. This is called the black hole problem. To prevent this, the base station mobility[8] is taken into consideration.

The optimal base station location is calculated by finding the distance from the cluster head at each feasible location. The minimum distance is optimal as the amount of energy required is minimum (distance is directly proportional to energy consumed in wireless transmission). The base station then moves to the location. Once the base station reaches the destination it sends a 'hello' packet to the cluster head.

\section{CONCLUSION}

The ultimate goal here is to maximize the network lifetime of a sensor network. Several approaches have been taken to achieve this goal. Firstly assumptions regarding the topology are made which simplifies the clustering phase of the network with heterogeneous nodes. The cluster head is chosen in each round as the highest energy node in the cluster unlike LEACH where the cluster head is randomly chosen. LEACH may choose a node with the least energy as the cluster head in the worst case. The distribution of budget not only considers the distance but also the energy factor of the node its allocating budget to. Multi path approach is used to guarantee an alternate path in order to maximize the network lifetime. Multi hop routing is used to transfer data from nodes which need not necessitate all nodes to be directly connected to the cluster head instead use intermediate nodes to transfer data to the cluster head. The mobility of the base stations avoids the black hole problem. This in turn increases the network lifetime.

\section{ACKNOWLEDGMENTS}

Our thanks to our professor Monica Mundada for her guidance and encouragement without which this would not have been possible.

\section{REFERENCES}

[1] Zheng Kai, Tong Libiao, Lu Wenjun Location-Based Routing Algorithms for Wireless Sensor Network - ZT communications, 2009

[2] Prof. Madhav Bokare1, Mrs. Anagha Ralegaonkar,Nanded. "Wireless Sensor Network:A Promising Approach for Distributed Sensing Tasks "Excel Journal of engineering technology and management science December-January 2012

[3] Vivek Katiyar, Narottam Chand, Surender Soni "Clustering Algorithms for Heterogeneous Wireless Sensor Network: A Survey"International journal of applied engineering research,Dindigul Volume 1,No 2,2010 .

[4] Rab Nawaz "'Cluster Based Routing for Wireless Sensor Network -Energy Efficient Cluster Based Routing Protocol for Wireless Sensor Networks", unpublished

[5] Monica R Mundada, Savan Kiran , Shivanand Khobanna1, Raja Nahusha Varsha and Seira Ann George "A study on Energy efficient routing protocols in wireless sensor networks" International Journal of Distributed and Parallel Systems (IJDPS) Vol.3, No.3, May 2012

[6] R Vidhyapriya , Dr P T Vanathi,"Energy Efficient Adaptive Multipath Routing for Wireless Sensor Networks",IAENG International Journal of computer science,34:1,IJCS_34_1_8

[7] Shashidhar Rao Gandham, Milind Dawande_, Ravi Prakash and S. Venkatesa " Energy Efficient Schemes for Wireless SensorNetworks with Multiple Mobile Base Stations" Global Telecommunications Conference, GLOBECOM . IEEE - GLOBECOM, 2003.

[8] A. P. Azad and A. Chockalingam "Mobile Base Stations Placement and Energy Aware Routing in Wireless Sensor Networks", WCNC 2006

[9] Hao Chen, Seapahn Megerian,"Cluster Sizing and Head Selection for Efficient Data Aggregation and Routing in Sensor Networks" WCNC 2006 IEEE,Volume 4,pages 2318-2323,Conference Publications.

[10] Razieh Sheikhpour and Sam Jabbehdari "An energy efficient cluster-chain based routing protocol for time criticalapplications in wireless sensor networks" Indian Journal of Science and Technology Vol.5 No.5, 2012. 